\title{
DESIGN DE GEMAS: IRRADIAÇÃO SELETIVA EM QUARTZO ROSA
}

Felipe Foerstnow Szczepaniak

Universidade Federal do Rio Grande do Sul - UFRGS

foerspak@gmail.com

Lauren da Cunha Duarte

Universidade Federal do Rio Grande do Sul - UFRGS

lauren.duarte@ufrgs.br

Pedro Luiz Juchem

Universidade Federal do Rio Grande do Sul - UFRGS

labogem@ufrgs.br

Fernando Soares Lameiras

Centro de Desenvolvimento da Tecnologia Nuclear - CDTN

fsl@cdtn.br

Resumo: Alguns materiais gemológicos são incolores ou possuem cores e tonalidades pouco atrativas, necessitando de tratamento para serem valorizados. A irradiação gama para alteração cromática de minerais é uma alternativa viável de tratamento para a valorização desses materiais, pois já é um tratamento reconhecido no comércio mundial. Este trabalho é resultado de uma pesquisa que teve como objetivo estudar parâmetros de irradiação gama seletiva em quartzo, um dos minerais mais abundantes da crosta terrestre, a fim de gerar contribuições aos profissionais que utilizam materiais gemológicos. Em amostras de quartzo rosa pálido, foi estudada a relação da espessura de placas de chumbo para atenuação da irradiação, contraste de tonalidade resultante da atenuação, largura de fresta entre as placas de chumbo e afastamento das amostras em relação às placas de chumbo.

Palavras-chave: design de gemas, quartzo, irradiação gama seletiva.

Abstract: Some gem materials are colorless or have unattractive colors and shades, and need treatment to be enhanced. Changes or improvement in color by gamma irradiation is a good choice and has been approved worldwide in the gemstone market This paper is the result of a research conducted to establish parameters of selective gamma irradiation on quartz, one of the most common mineral in the earth's crust, in order to generate contributions to develop unique gemological materials. In samples of pale pink quartz, it was studied the relation between the thickness of lead plates to irradiation attenuation, the final color tone contrast obtained by 
attenuation, slot spacing between the lead samples and the samples distance from the lead in relation to the irradiation attenuation.

Keywords: gems design, quartz, selective gamma irradiation.

\section{INTRODUÇÃO}

Dentre os minerais mais comuns do planeta está o quartzo, de fórmula química $\mathrm{SiO}_{2}$, conforme Frondel (1962) e Anderson (1984), que pode ser encontrado em diferentes variedades gemológicas, incluindo gemas límpidas, incolores e transparentes (cristal de rocha) ou gemas com texturas visuais coloridas como ametista (violeta), citrino (amarelo) e quartzo rosa. As variedades incolores e algumas coloridas, podem ser submetidas à irradiação gama homogênea e seletiva com ${ }^{60} \mathrm{Co}$, resultando em diferentes colorações. Essas novas colorações e bicolorações são importantes elementos para o design de gemas, segundo Alkmim (2012), Nejeliski et al. (2014), Szczepaniak et al. (2014) e Szczepaniak (2016).

A irradiação gemológica é normalmente feita de modo homogêneo, porém, a irradiação seletiva, com o bloqueio ou atenuação da irradiação, significa expor somente partes de um objeto à fonte de irradiação e outras partes protegidas com blindagens. É comumente usada em exames médicos de raios $X$, quando partes do corpo humano são protegidas da radiação, conforme Tauhata et al. (2014). De modo geral, na irradiação seletiva pode-se considerar: 1) a atenuação da irradiação por algum material, como por exemplo, o estudo de Korkut et al. (2010) que utiliza a ametista como material atenuante da irradiação e 2) a passagem da irradiação por máscaras, frestas e orifícios, dentre outros, como por exemplo, o estudo de Novaes e Brito (2007) que trata da irradiação seletiva em camundongos.

Objetiva-se nesta pesquisa estudar parâmetros do processo de irradiação gama seletiva no quartzo rosa pálido de Pedra Lavrada, PB, a fim de gerar parâmetros que possam ser aplicados para o desenvolvimento de grafismo em gemas.

\section{DESENVOLVIMENTO}

No desenvolvimento desta pesquisa, foram utilizados os materiais e métodos a seguir descritos.

Foram utilizadas amostras de quartzo rosa pálido muito límpidas, doadas pelo geólogo Sandro Ferreira. Elas são provenientes do Alto do Feio localizado na cidade de Pedra Lavrada, PB.

Antes de iniciar a irradiação seletiva, foram irradiadas amostras de modo homogêneo para verificação das intensidades das doses necessárias para haver mudança de cor e da homogeneidade da dose. Para os estudos da irradiação seletiva, foram preparadas amostras polidas com aproximadamente $30 \mathrm{~mm}$ de comprimento e $4 \mathrm{~mm}$ de largura e de espessura.

Para atenuar a irradiação gama, foram utilizadas sete placas padronizadas de chumbo com $99,97 \%$ de pureza e densidade $11,34 \mathrm{~g} / \mathrm{cm}^{3}$, conforme norma ASTM B29 (American Society for Testing \& Materials). As amostras possuem a forma de prismas retangulares (superfícies planas e paralelas), com $10 \mathrm{~cm}$ de comprimento e de largura, e espessura de $5 \mathrm{~cm}$; apenas uma das amostras possui a espessura diferenciada, com 3 
$\mathrm{cm}$. Com essas placas consegue-se as espessuras de $3 \mathrm{~cm}$ e $5 \mathrm{~cm}$ e por justaposição de duas placas, espessuras de $10 \mathrm{~cm}$ e de $20 \mathrm{~cm}$.

Neste trabalho, as amostras de quartzo rosa pálido foram irradiadas no Laboratório de Irradiação Gama - LIG do Centro de Desenvolvimento da Tecnologia Nuclear - CDTN. O LIG é equipado com um Irradiador Panorâmico Múltipropósito (Figura 1), provido de fonte de ${ }^{60} \mathrm{Co}$ estocada a seco, onde foram executadas as irradiações homogêneas e as seletivas. As doses de irradiação são representadas em gray (Gy), que expressa a quantidade de energia absorvida pela matéria por unidade de massa ( $1 \mathrm{~Gy}=1$ joule de energia absorvido por $1 \mathrm{~kg}$ de matéria; $1 \mathrm{kGy}=1.000 \mathrm{~Gy}$ ).

A distância das amostras testadas à fonte foi estabelecida a partir do experimento de Andrade (2014), resultando na padronização de $30 \mathrm{~cm}$ de distância e alturas (das amostras ao piso) de $43 \mathrm{~cm}$ e aproximadamente $57,5 \mathrm{~cm}$ (centro da fonte). Utilizaramse as placas de chumbo como atenuadores da irradiação e fita adesiva Eurocel $^{\circledR}$ Ecopack 10 entre o chumbo e as amostras de quartzo, para não contaminar as amostras estudadas com o chumbo, pois elas devem permanecer encostadas nas placas.

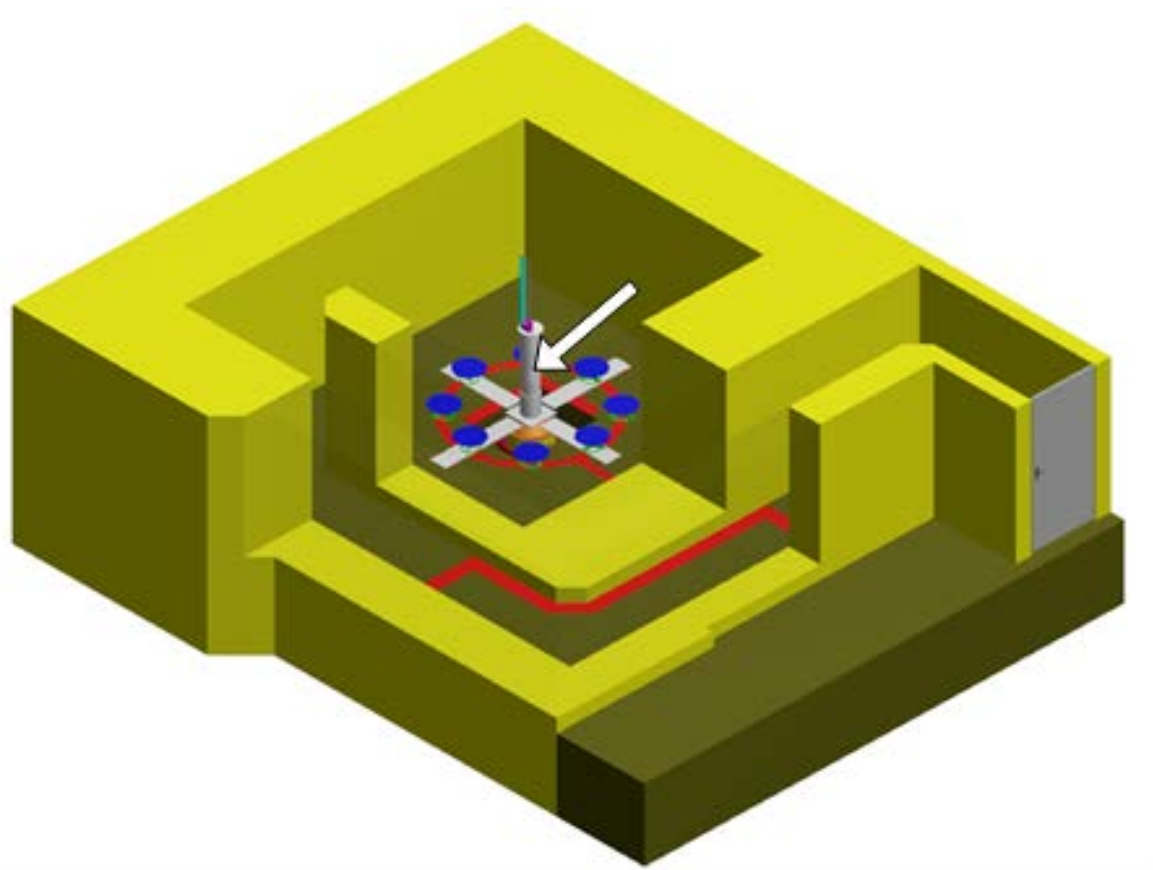

Figura 1 - Maquete eletrônica da câmara de irradiação do LIG expondo a fonte de ${ }^{60}$ Co centralizada (seta).

Fonte: Adaptado de http://www.cdtn.br/linhas_de_atuacao/Laboratorios/Visao3D daCamaradelrradiacaoBlindagemBiologicaeLabirintodoLIG.pdf.

Foram utilizadas quatro espessuras de placas de chumbo como atenuação para a blindagem da irradiação gama, de $3 \mathrm{~cm}, 5 \mathrm{~cm}, 10 \mathrm{~cm}$ e $20 \mathrm{~cm}$. As doses selecionadas de irradiação gama foram de 5 kGy, 10 kGy e 15 kGy, que correspondem às doses que geraram tonalidades cinza acastanhadas na irradiação homogênea. A distância entre duas placas de chumbo, aqui denominadas de frestas, foram determinadas a partir de testes anteriores, como sendo de $1 \mathrm{~mm} 3 \mathrm{~mm}$ e $5 \mathrm{~mm}$. Além desse experimento, deixa-se aproximadamente $4 \mathrm{~mm}$ das amostras de quartzo expostas a irradiação, sendo as outras partes preservadas pela sobreposição das placas de chumbo. Com relação aos testes com fresta, deixa-se a parte central das amostras em exposição 
(Figura 2). As frestas laterais geradas pela espessura das amostras de quartzo foram cobertas por outras amostras de chumbo, deixando somente a parte frontal ou superior das amostras de quartzo em exposição. Em cada experimento de irradiação foram utilizadas três amostras de quartzo e uma de vidro, como amostra-controle.

O quartzo irradiado foi fotografado com câmera digital Sony Cyber-shot, modelo DSC-W210, junto à legenda de cores (RGB e CMYK) para auxiliar a observação dos resultados em diferentes meios de comunicação (digital e impresso).
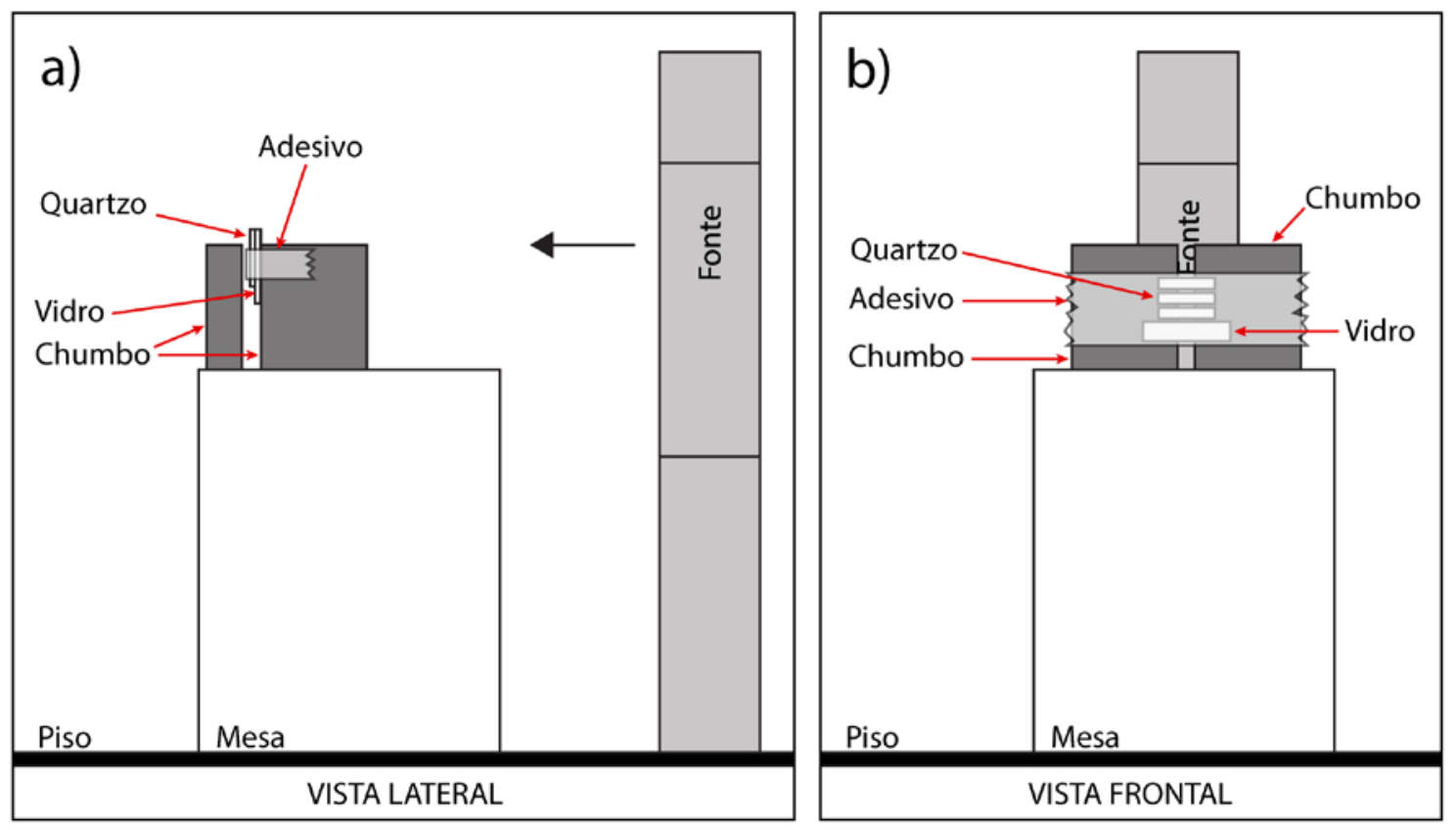

Figura 2 - Esquema simplificado da irradiação gama seletiva utilizada na câmara do LIG. a) vista lateral da irradiação das extremidades superiores das amostras de quartzo blindadas com chumbo e b) vista frontal da irradiação seletiva em quartzo com fresta entre as duas placas de chumbo.

Fonte: Elaborado pelos autores, com base na pesquisa realizada.

\subsection{Resultados e discussão}

Todas as amostras de quartzo irradiadas de forma homogênea apresentaram alteração cromática para o matiz cinza acastanhado em diferentes tonalidades, e estão de acordo com os estudos de Miranda et al. (2012). Segundo Rossman (1994), Nassau (2001) e Lameiras et al. (2006), o escurecimento do quartzo é devido as impurezas de alumínio comuns no quartzo, apesar de ocorrer em teores baixos (80 a 100 ppm). Conforme as doses de irradiação gama foram aumentadas, as amostras de quartzo rosa-pálido estudadas escureceram.

Em sequência, são mostrados e descritos os principais resultados da Irradiação gama seletiva no quartzo rosa pálido de Pedra Lavrada, constituindo testes básicos necessários para a área de design de gemas. Os resultados obtidos com diferentes doses de irradiação e da atenuação dessa irradiação utilizando diferentes espessuras de placas de chumbo, foram nomeados de " $a$ " a " $h$ " e são mostrados nas figuras abaixo (Figuras 3 a 6). Tendo em vista as infinitas possibilidades de uso da irradiação gama seletiva e o gosto pessoal do projetista, não são gerados parâmetros definitivos e restrições, mas apenas algumas indicações.

A Figura 3 mostra os resultados obtidos utilizando-se placas de chumbo de 20 $\mathrm{cm}$ para atenuação da irradiação, com a seguinte variação das doses de irradiação: 5 
kGy (Figura 3a); 10 kGy (Figura 3b); 15 kGy (Figura 3c). $43 \mathrm{~cm}$ é a altura do experimento em relação ao piso. Observa-se claramente que houve um aumento na tonalidade da cor cinza acastanhada com o aumento da dose de irradiação. Nesse experimento, dentre essas doses utilizadas, indica-se a dose de $10 \mathrm{kGy}$, por manter boa tonalidade de cor, pois não está escura demais, permitindo boa transparência.

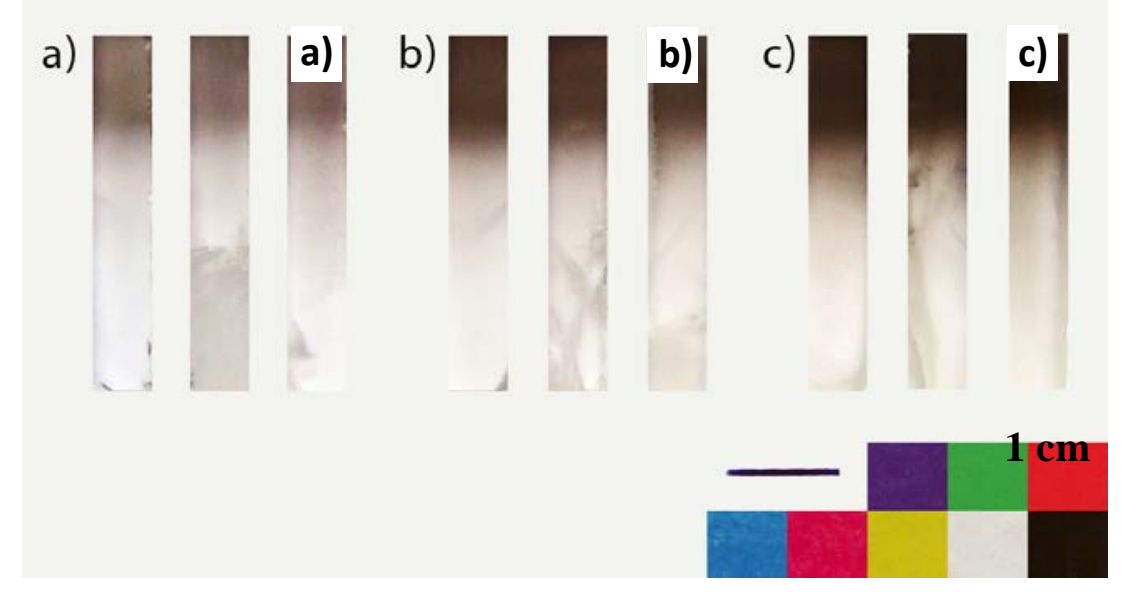

Figura 3 - Resultados (detalhes) das irradiações seletivas no quartzo-rosa pálido de Pedra Lavrada, utilizando-se placas de chumbo de $20 \mathrm{~cm}$ para atenuação da irradiação. Amostras com $4 \mathrm{~mm}$ de espessura e irradiadas com diferentes doses: a) $\mathbf{5}$ kGy, b) 10 kGy e c) 15 kGy.

Fonte: Elaborado pelos autores, com base na pesquisa realizada.

A Figura 4 mostra os resultados obtidos utilizando-se sempre a mesma dose de irradiação (10 kGy), variando-se a espessura das placas de chumbo para atenuação da irradiação: $10 \mathrm{~cm}$ de espessura (Figura 4d); $5 \mathrm{~cm}$ de espessura (Figura 4e); $3 \mathrm{~cm}$ de espessura (Figura 4f). A altura do experimento em relação ao piso é de $43 \mathrm{~cm}$. Observa-se que as amostras das Figuras $4 \mathrm{e}$ e $4 \mathrm{f}$ apresentam pouco contraste.

Observa-se que nesses experimentos de irradiação seletiva houve forte atenuação da irradiação gama promovida pelo chumbo (Figuras 3 e 4d), resultando em amostras com alto contraste de cor, o que é um recurso de grande valia para o Design. Com relação à placa de chumbo para atenuação, indica-se a espessura de $10 \mathrm{~cm}$ e menores que $10 \mathrm{~cm}$, levando em consideração, no entanto, que valores menores resultarão em menor contraste, colorindo toda a amostra, mostrando menor contraste. A relação entre a dose de $10 \mathrm{kGy}$ com $3 \mathrm{~cm}$ de espessura da placa de chumbo para atenuação apresentou baixíssimo contraste (Figura 4f), o que pode ser interessante para alguns projetos. Já a relação da dose de 10 kGy com atenuação de $5 \mathrm{~cm}$ de espessura da placa de chumbo, apresentou baixo contraste (Figura 4e), pois coloriu a parte blindada da gema com pouca intensidade, não deixando incolor essa parte. Não foi notada alteração de cor na parte blindada das amostras de quartzo onde foram utilizadas placas de chumbo para atenuação com $20 \mathrm{~cm}$ (Figura 3) e $10 \mathrm{~cm}$ de espessura (Figura 4d), apresentando alto contraste da parte irradiada em relação à parte blindada. 


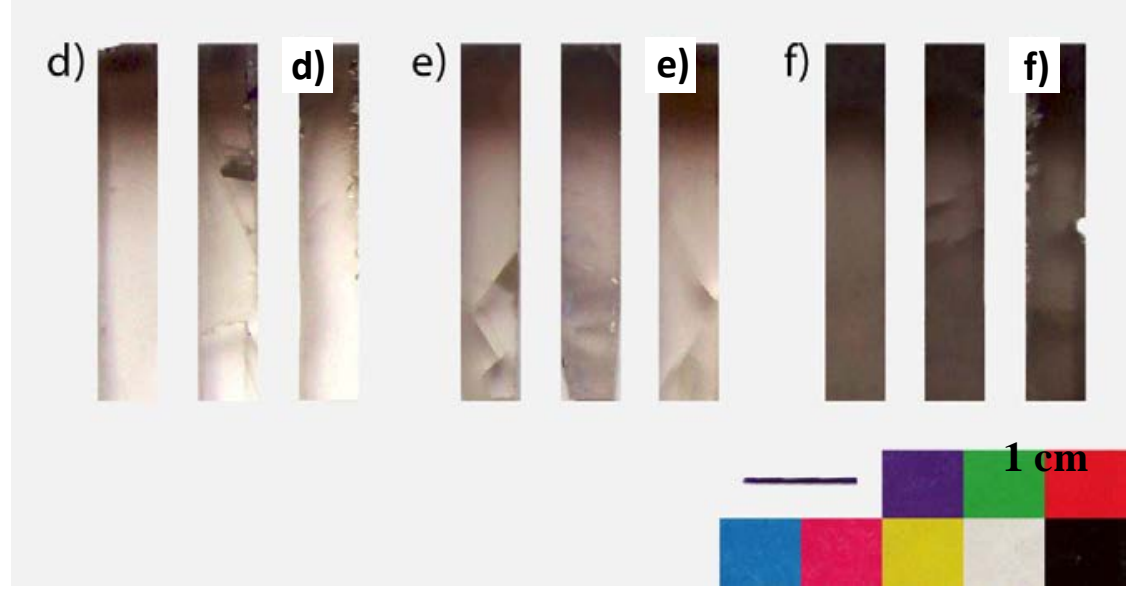

Figura 4 - Resultados (detalhes) das irradiações seletivas no quartzo-rosa pálido de Pedra Lavrada, utilizando-se sempre a mesma dose de irradiação: $10 \mathrm{kGy}$. Amostras com $4 \mathrm{~mm}$ de espessura e irradiadas com placas de chumbo com diferentes espessuras para atenuar a irradiação: d) atenuação de $10 \mathrm{~cm}$ de chumbo, e) atenuação de $5 \mathrm{~cm}$ de chumbo e f) atenuação de $\mathbf{3} \mathrm{cm}$ de chumbo.

Fonte: Elaborado pelos autores, com base na pesquisa realizada.

A Figura 5 mostra o resultado obtido com a variação da largura das frestas entre as chapas de chumbo, utilizando-se a dose de irradiação de 10 kGy, espessura de $10 \mathrm{~cm}$ da placa de chumbo para atenuar a irradiação e altura do experimento em relação ao piso de aproximadamente $57,5 \mathrm{~cm}$ : fresta com $5 \mathrm{~mm}$ (Figura $5 \mathrm{~g}$ ); fresta com $3 \mathrm{~mm}$ (Figura 5h); fresta com $1 \mathrm{~mm}$. Observa-se que houve uma redução na intensidade da cor das amostras conforme a diminuição das frestas ( $3 \mathrm{~mm}$ e $5 \mathrm{~mm}$ ) e, utilizando-se $1 \mathrm{~mm}$ de fresta (experimento não mostrado) não se pôde observar coloração resultante. Esses resultados alcançados corresponderam à expectativa gerada na pesquisa do Wu et al. (1990), embora esses autores tenham utilizado outras configurações experimentais.

h)

g)

h)

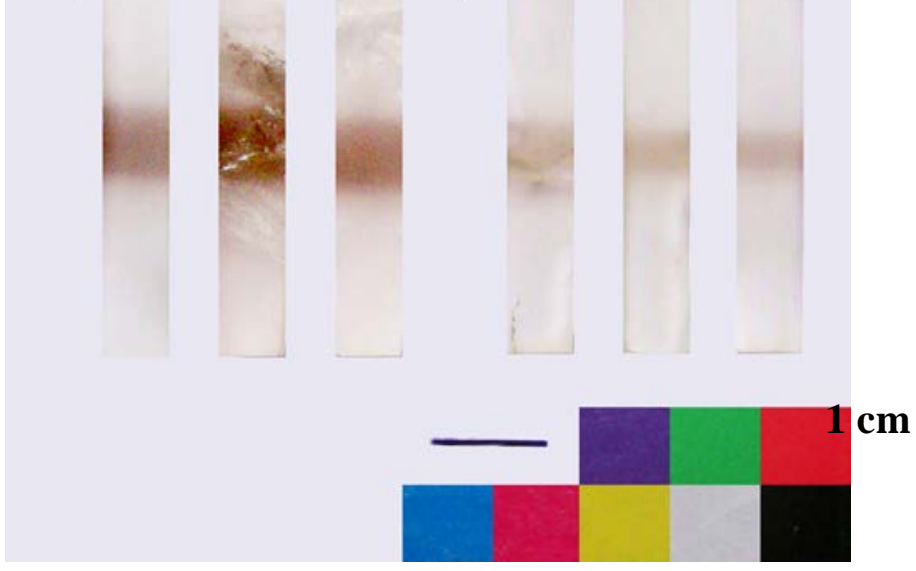

Figura 5 - Resultados (detalhes) das irradiações seletivas no quartzo rosa-pálido de Pedra Lavrada, utilizando-se a dose de irradiação de 10 kGy e atenuação da irradiação com placa de chumbo de $10 \mathrm{~cm}$ de espessura. Amostras com $4 \mathrm{~mm}$ de espessura, polidas e irradiadas com diferentes espaçamentos de frestas: g) $5 \mathbf{m m}$ de espaçamento de fresta e h) $\mathbf{3} \mathbf{m m}$ de espaçamento de fresta.

Fonte: Elaborado pelos autores, com base na pesquisa realizada. 
Foi realizado também um experimento variando a distância entre a placa de chumbo e a amostra, utilizando-se os seguintes parâmetros: dose de 10 kGy, atenuação com $10 \mathrm{~cm}$ de espessura de chumbo e afastamento de $6 \mathrm{~mm}$ da amostra em relação ao chumbo. Esse afastamento foi realizado com uma folha de papel dobrada, resultando em uma forma volumétrica semelhante a um paralelepípedo oco. Esse experimento, teve por finalidade a validação dos experimentos anteriores, pois em todos eles foi colocado apenas fita adesiva entre as amostras de quartzo e o chumbo, deixando o quartzo a mícrons de distância do chumbo, praticamente encostado nas placas de atenuação da irradiação. Como resultado, o afastamento maior não expôs diferença visível em relação aos outros experimentos com as amostras encostadas no chumbo, indicando que para a irradiação podem ser utilizadas gemas brutas irregulares e outros materiais de proteção no chumbo.

\section{CONCLUSÃO}

A irradiação gama seletiva é um processo de beneficiamento polêmico (por causa do uso de radiação ionizante) e trabalhoso, mas tecnicamente viável, podendo ser realizados trabalhos exclusivos com poucas reproduções, embora possam ser também concebidos trabalhos padronizados em série. Os recursos de atenuação, blindagem e sobreposição de doses permitiram coletar dados importantes para o design de gemas.

O material gemológico quartzo rosa pálido foi de suma importância para este estudo, por causa da sua transparência, poucas fraturas e sensibilidade à irradiação.

Os principais parâmetros de irradiação gama seletiva obtidos para o quartzo rosa estudado são: 1) Dose de $10 \mathrm{kGy}$ para espessura de $4 \mathrm{~mm}$ do quartzo (pode ser lapidado); 2) Atenuação de $10 \mathrm{~cm}$ de espessura da placa de chumbo, ou menor (até 3 $\mathrm{cm})$; 3) As amostras devem ser irradiadas com $30 \mathrm{~cm}$ de distância da fonte e 4) A fresta entre as placas de chumbo deve ter intervalos $\geq 3 \mathrm{~mm}$ de largura.

Os dados obtidos são importantes para futuras pesquisas sobre "design de superfície" em materiais gemológicos, principalmente quanto ao propósito de desenvolver desenhos com tal procedimento. No entanto, é necessário o estudo da usinagem em diferentes materiais atenuadores de radiação para desenvolver os desenhos. Ressalta-se que a usinagem do chumbo pode ser perigosa e com dificuldade para a sua realização, pois diversos locais se recusam a trabalhar com este metal devido à sua toxidade quando aspirado pelo corpo humano. Recomenda-se o CDTN para a correta usinagem do chumbo - principalmente com relação ao descarte do material tóxico.

\section{AGRADECIMENTOS}

Luiz Carlos Duarte Ladeira, Fernando Soares Lameiras, Márcio Pereira, Maritza Gual, Helena Cristina de Matos Silva, Cleide Rocha, Lorrane Goveia, Antônio Timóteo, Omar, Sandro Ferreira, CAPES e Projeto Universal do CNPq.

\section{REFERÊNCIAS}

ALKMIM, D. G. Identificação do potencial de desenvolvimento de cor em quartzo natural incolor por meio da espectrometria Raman. 2012. Dissertação (mestrado) - 
Centro de Desenvolvimento da Tecnologia Nuclear, Programa de Pós-graduação em Ciência e Tecnologia das Radiações, Minerais e Materiais.

ANDERSON, B. W. A identificação das gemas. Rio de Janeiro: Ao Livro Técnico, 1984.

ANDRADE, M. F. Comparação dos efeitos da terapia laser de baixa intensidade (lambda $=660 \mathrm{~nm}$ ou lambda $\mathbf{7 8 0} \mathrm{nm}$ ) no tratamento de mucosite oral induzida por radiação ionizante em ratos. 2014. Dissertação (mestrado) - Autarquia associada à Universidade de São Paulo, Instituto de Pesquisas Energéticas e Nucleares.

CDTN - Centro de Desenvolvimento da Tecnologia Nuclear. O CDTN. Disponível em: <http://www.cdtn.br/O_CDTN> Acesso em: 12 nov. 2014.

FRONDEL, C. The System of Mineralogy of James Dwight Dana and Edward Salisbury Dana. v. 3, Silica Minerals, 7th ed., New York and London: John Wiley \& Sons, 1962.

KORKUT, T.; KORKUT, H.; KARABULUT, A.; BUDAK, G. A new radiation shielding material: amethyst ore. Annals of Nuclear Energy. P.56-59, 2010.

LAMEIRAS, F. S.; NUNES, E. H. M.; MELO, V. A. R.; LIZ, O. R. Identificação de quartzos incolores para joalheria. REM: R. Esc. Minas, Ouro Preto, 59(1), p. 129-133, jan., mar., 2006.

LIG - Laboratório de Irradiação Gama do Centro de Desenvolvimento da Tecnologia Nuclear - CDTN. Catálogo. Laboratório de Irradiação Gama - LIG, Centro de Desenvolvimento da Tecnologia Nuclear - CDTN, s/d.

MIRANDA, M. R.; GONZAGA, R. S. G.; GUZZO, P. L.; BARRETO, S. B.; MELGAREJO, J. C. Estudo da suscetibilidade ao escurecimento por radiação gama de quartzo rózeoleitoso da província pegmatítica da Borborema. Revista Brasileira de Geociências, vol. 42 (2), 2012.

NEJELISKI, D. M.; SZCZEPANIAK, F. F.; DUARTE, L. C.; ROLDO, L. Materiais e design: compósito de moscovita e vidro. In: 11을 Congresso Brasileiro de Pesquisa e Desenvolvimento em Design 2014. Anais do 110 Congresso Brasileiro de Pesquisa e Desenvolvimento em Design. São Paulo: Editora Edgard Blücher, p. 2182-2193, 2014.

NASSAU, K. The physics and chemistry of color: the fifteen causes of color. New York: John Wiley, 2001.

NOVAES E BRITO, R. R.. Irradiação ionizante seletiva impede a evolução do lupus eritematoso sistemico em camundongo fêmeo (NZB/NZW) F1 e seleciona população radiorresistente de células B-1 peritoneais. 2007. Tese (doutorado) - Universidade Federal de São Paulo.

ROSSMAN, G. R. The colored varieties of silica. Reviews in Mineralogy 29, p. 433-68, 1994.

SZCZEPANIAK, F. F. Irradiação gama $\left({ }^{60} \mathrm{Co}\right)$ homogênea e seletiva em quartzo rosa visando o design de materiais gemológicos. 2016. Dissertação (mestrado) Universidade Federal do Rio Grande do Sul, Curso de Pós-Graduação em Design e Tecnologia.

SZCZEPANIAK, F. F.; DUARTE, L. C.; JUCHEM, P. L. Design de superfície de gemas: simulação do quartzo rutilado através da metalização de ouro em estêncil. In: 
HARTMANN, L. A. Tecnologia e inovação em gemas, joias e mineração. Porto Alegre: IGEO/UFRGS, 2014.

TAUHATA, L.; SALATI, I.; DI PRINZIO, R.; DI PRINZIO, A. R. Radioproteção e dosimetria: fundamentos. 10a ed. Rio de Janeiro: IRD/CNEN, 2014.

TILLY JUNIOR, J. G. Física radiológica. Rio de Janeiro: Guanabara Koogan, 2010.

WU, A.; LINDNER, G.; MAITZ, A. H.; KALEND, A. M.; LUNSFORD, L. D.; FLICKINGER, J. C.; BLOOMER, W. D. Physics of Gamma Knife approach on convergent beams in stereotactic radiosurgery. I. J. Radiation Oncology - Biology - Physics. vol. 18, n. 4, abril, p. 941-949, 1990. 